International Journal of Pure and Applied Mathematics

Volume 107 No. 3 2016, 625-634

ISSN: 1311-8080 (printed version); ISSN: 1314-3395 (on-line version)

url: http://www.ijpam.eu

doi: 10.12732/ijpam.v107i3.11

\title{
SKEW N-NORMAL COMPOSITION AND WEIGHTED COMPOSITION OPERATORS ON $L^{2}(\mu)$
}

\author{
Anuradha Gupta ${ }^{1}$, Renu Chugh ${ }^{2}$, Jagjeet Jakhar ${ }^{3 \S}$ \\ ${ }^{1}$ Department of Mathematics \\ Delhi College of Arts and Commerce \\ University of Delhi \\ Delhi, 110023, INDIA \\ ${ }^{2,3}$ Department of Mathematics \\ M.D. University \\ Rohtak, 124001, Haryana, INDIA
}

\begin{abstract}
An operator $T$ is called skew n-normal operator if $\left(T^{\mathrm{n}} T^{*}\right) T=T\left(T^{*} T^{\mathrm{n}}\right)$, for all natural number $\mathrm{n}$. In this paper, the condition under which composition operators and weighted composition operators become skew n-normal operators have been obtained in terms of radon-nikodym derivative $h_{\mathrm{n}}$. We investigate some basic properties of such operators and study the relation among non normal composition operators and the skew n-normal composition operators.
\end{abstract}

AMS Subject Classification: 47B33, 47B20, 46C05

Key Words: composition operators, weighted composition operators, normal operator, quasi-normal operator, n-normal operator, skew n-normal operator

\section{Introduction}

Let $H$ be the infinite dimensional complex Hilbert space and $B(H)$ be the algebra of all bounded linear operators on $H$. An operator $T \in B(H)$ is said to be self-adjoint (see [3]) if $T^{*}=T$, normal (see [2]) if $T T^{*}=T^{*} T$, quasinormal (see [11]) if $T\left(T^{*} T\right)=\left(T^{*} T\right) T$. An operator $T$ is called n-normal (see $\left.[2,6]\right)$ if

Received: January 18, 2016

Published: April 21, 2016

§ Correspondence author (c) 2016 Academic Publications, Ltd.

url: www.acadpubl.eu 
$T^{n} T^{*}=T^{*} T^{n}$, n-quasinormal operator (see $\left.[1,9]\right)$ if $T^{n} T^{*} T=T^{*} T T^{n}$, skew n-normal operators (see [10]) if $\left(T^{n} T^{*}\right) T=T\left(T^{*} T^{n}\right)$ for $n \in N$.

Let $(X \mathcal{A}, \mu)$ be a $\sigma$-finite measure space. A transformation $T$ is said to be measurable if $T^{-1}(B) \in \mathcal{A}$ for $B \in \mathcal{A}$. A measurable transformation $T$ is said to be non-singular if

$$
\mu\left(T^{-1}(B)\right)=0 \text { whenever } \mu(B)=0 \text { for every } B \in \mathcal{A} .
$$

If $T$ is a measurable transformation then $T^{n}$ is also a measurable transformation, for all natural number n. If $T$ is non-singular, then we say that $\mu\left(T^{-1}\right)$ is absolutely continuous with respect to $\mu$ and hence $\mu\left(T^{-1}\right)^{n}$ becomes absolutely continuous with respect to $\mu$. Hence, by Radon-Nikodym theorem there exists a unique non-negative essentially bounded measurable function $h_{n}$ such that

$$
\mu\left(T^{-1}\right)^{n}(B)=\int_{B} h_{n} d \mu \quad \text { for } B \in \mathcal{A}
$$

and $h_{n}$ is called the Radon-Nikodym derivative and is denoted by $\frac{d \mu\left(T^{-1}\right)^{\mathrm{n}}}{d \mu}$.

Let $\phi$ be an essentially bounded function. The multiplication operator $M_{\phi}$ on the space $L^{2}(\mu)$ induced by $\phi$ is given by

$$
M_{\phi} f=\phi f \quad \text { for } f \in L^{2}(\mu)
$$

Let $T$ be a measurable transformation on $X$. The composition operator $C_{T}$ on the space $L^{2}(\mu)$ is given by

$$
C_{T} f=f \circ T \quad \text { for } f \in L^{2}(\mu)
$$

Let $\phi$ be a complex-valued measurable function. The weighted composition operator $W_{\phi, T}$ on the space $L^{2}(\mu)$ induced by $\phi$ and $T$ is given by

$$
W_{\phi, T} f=\phi . f \circ T \quad \text { for } f \in L^{2}(\mu) .
$$

For more on composition operators and weighted composition operators one may refer to $[4-8,12]$.

Proposition 1. Change of Variables: Let $X$ be a non-empty set and let $\mathcal{A}$ be a $\sigma$-algebra on $X$. Let $\mu$ and $\mu T^{-1}$ be measures on $\mathcal{A}$ and let $h: X \rightarrow[0,1]$ be a measurable function. Then the following are equivalent:

(i) $\mu T^{-1}$ is absolutely continuous with respect to $\mu$ and $h$ is Radon-Nikodym derivative of $\mu T^{-1}$ with respect to $\mu$.

(ii) For every measurable function $f: X \rightarrow[0 ; 1]$, the equality

$$
\int_{X} f d \mu T^{-1}=\int_{X} f h d \mu
$$


holds.

The conditional expectation operator $E\left(. \mid T^{-1}(\mathcal{A})\right)=E(f)$ is defined for each non-negative function $f$ in $L^{p}(1 \leq p<\infty)$ and is uniquely determined by the following set of conditions:

(i) $E(f)$ is $T^{-1}(\mathcal{A})$ measurable. have

(ii) If $B$ is any $T^{-1}(\mathcal{A})$ measurable set for which $\int_{B} f d \mu$ converges then we

$$
\int_{B} f d \mu=\int_{B} E(f) d \mu .
$$

The conditional expectation operator $E$ has the following properties:

(i) $E(f . g \circ T)=(E(f))(g \circ T)$.

(ii) $E$ is monotonically increasing, i.e., if $f \leq g$ a.e. then $E(f) \leq E(g)$ a.e.

(iii) $E(1)=1$.

(iv) $E(f)$ has the form $E(f)=g \circ T$ for exactly one $\mathcal{A}$-measurable function $g$ provided that the support of $g$ lies in the support of $h$ which is given by

$$
\sigma(h)=\{x: h(x) \neq 0\} .
$$

$E$ is the projection operator onto the closure of the range of the composition operator $C_{T}$ on $L^{2}(\mu)$.

Motivated by the approach and direction of research by the mathematicians in $[4,5,6,9,10]$, an effort has been made in the paper to discuss the behaviour of skew n-normal class of operators. In this paper, we study skew n-normal composition operators and weighted composition operators in terms of RadonNikodym derivative and expectation operators.

\section{Skew n-Normal Composition Operators}

Let $C_{T}$ be the composition operator and $C_{T}^{*}$ be its adjoint which is given by $C_{T}^{*} f=h . E(f) \circ T^{-1}$.

Lemma 2. $[8,12]$ Let $P$ be the projection on $L^{2}(X, \mathcal{A}, \mu)$ onto $\overline{R\left(C_{T}\right)}$. Then:

(i) $C_{T}^{*} C_{T} f=h f$ and $C_{T} C_{T}^{*} f=(h \circ T) P f$ for all $f \in L^{2}(\mu)$.

(ii) $\overline{R\left(C_{T}\right)}=\left\{f \in L^{2}(\mu): f \quad\right.$ is $\left.\quad T^{-1}(\mathcal{A})\right\}$ measurable. 
(iii) If $f$ is $T^{-1}(\mathcal{A})$ measurable and $g$ and $f g$ belong to $L^{2}(\mu)$, then $P(f g)=$ $f P(g)$, ( $f$ need not be in $\left.L^{2}(\mu)\right)$.

(iv) $\left(C_{T}^{*} C_{T}\right)^{k} f=h^{k} f$ for all $k \in \mathcal{N}$.

(v) $\left(C_{T} C_{T}^{*}\right)^{k} f=(h \circ T)^{k} P(f)$.

(vi) $E$ is the identity operator on $L^{2}(\mu)$ if and if $T^{-1}(\mathcal{A})=\mathcal{A}$.

Theorem 3. Let $C_{T}$ be a composition operator on $L^{2}(\mu)$. Then the following statements are equivalent:

(i) $C_{T}$ is skew n-normal operator.

(ii) $h \circ T^{n}=h \circ T$.

Proof. For $f \in L^{2}(\mu)$

$$
\begin{aligned}
\left(C_{T}^{n} C_{T}^{*}\right) C_{T} f & =\left(C_{T}^{n} C_{T}^{*}\right) f \circ T \\
& =C_{T}^{n}\left(h \cdot E(f \circ T) \circ T^{-1}\right) \\
& =\left(h \cdot E(f \circ T) \circ T^{-1}\right) \circ T^{n} \\
& =h \circ T^{n} \cdot E\left(f \circ T^{n}\right) .
\end{aligned}
$$

Also,

$$
\begin{aligned}
C_{T}\left(C_{T}^{*} C_{T}^{n}\right) f & =C_{T} C_{T}^{*}\left(f \circ T^{n}\right) \\
& =C_{T} h \cdot E\left(f \circ T^{n}\right) \circ T^{-1} \\
& =h \cdot E\left(f \circ T^{n-1}\right) \circ T \\
& =h \circ T \cdot\left(E\left(f \circ T^{n}\right)\right) .
\end{aligned}
$$

If $C_{T}$ is skew n-normal operator then

$$
\left(C_{T}^{n} C_{T}^{*}\right) C_{T}=C_{T}\left(C_{T}^{*} C_{T}^{n}\right) \Longleftrightarrow h \circ T^{n}=h \circ T .
$$

Corollary 4. Let $C_{T}$ be a composition operator on $L^{2}(\mu)$. then the following statements are equivalent:

(i) $C_{T}$ is skew n-normal operator.

(ii) $\left\|\sqrt{h \circ T^{n}}\right\|=\|\sqrt{h \circ T}\|$.

Corollary 5. An operator $C_{T}$ is skew n-normal operator iff $C_{T}^{*}$ is skew n-normal operator. 
Proof. Let $C_{T}$ be a skew n-normal operator therefore

$$
\left(C_{T}^{n} C_{T}^{*}\right) C_{T}=C_{T}\left(C_{T}^{*} C_{T}^{n}\right)
$$

Taking adjoint on both side, we have

$$
C_{T}^{*}\left(C_{T} C_{T}^{* n}\right)=\left(C_{T}^{* n} C_{T}\right) C_{T}^{*}
$$

Therefore $C_{T}^{*}$ is skew n-normal operator.

In this theorem we explain the condition under which the adjoint of $C_{T}$ is skew n-normal operator.

Theorem 6. An operator $C_{T}^{*} \in L^{2}(\mu)$ is skew n-normal operator iff

$$
h_{n} \cdot h=h_{n} \cdot h \circ T^{-n+1}
$$

Proof. Suppose $C_{T}^{*}$ is skew n-normal operator. Since

$$
C_{T}^{*}\left(C_{T} C_{T}^{* n}\right)=\left(C_{T}^{* n} C_{T}\right) C_{T}^{*}
$$

We have

$$
\begin{aligned}
C_{T}^{*}\left(C_{T} C_{T}^{* n}\right) f & =C_{T}^{*} C_{T}\left(h_{n} \cdot E(f) \circ T^{-n}\right) \\
& =C_{T}^{*}\left(h_{n} \cdot E(f) \circ T^{-n} \circ T\right) \\
& =h E\left(h_{n} \circ T E(f) \circ T^{1-n}\right) \circ T^{-1} \\
& =h E\left(h_{n} \cdot E(f) \circ T^{-n}\right) \\
& =h \cdot h_{n} f \circ T^{-n} .
\end{aligned}
$$

Also,

$$
\begin{aligned}
\left(C_{T}^{* n} C_{T}\right) C_{T}^{*} f & =C_{T}^{* n} C_{T}\left(h \cdot E(f) \circ T^{-1}\right) \\
& =C_{T}^{* n}\left(h \circ T \cdot E(f) \circ T^{-1} \circ T\right) \\
& =h_{n}\left(h \circ T \cdot E(f) \circ T^{-n}\right) \\
& =h_{n} h \circ T^{-n+1} f \circ T^{-n} .
\end{aligned}
$$

If $C_{T}^{*}$ is skew n-normal operator then

$$
C_{T}^{*}\left(C_{T} C_{T}^{* n}\right)=\left(C_{T}^{* n} C_{T}\right) C_{T}^{*} \Longleftrightarrow h_{n} . h=h_{n} . h \circ T^{-n+1} .
$$

Theorem 7. If $C_{T}$ is skew n-normal operator on $L^{2}(\mu)$. Then $\alpha C_{T}$ is skew n-normal operator for every complex number $\alpha$. 
Proof. Consider

$$
\begin{aligned}
\left(\left(\alpha C_{T}\right)^{n}\left(\alpha C_{T}\right)^{*}\right)\left(\alpha C_{T}\right) & =\alpha^{n} \bar{\alpha} \alpha\left(C_{T}^{n} C_{T}^{*}\right) C_{T} \\
& =\alpha^{n} \bar{\alpha} \alpha C_{T}\left(C_{T}^{*} C_{T}^{n}\right) \\
& =\left(\alpha C_{T}\right)\left(\left(\alpha C_{T}\right)^{*}\left(\alpha C_{T}\right)^{n}\right)
\end{aligned}
$$

so that $\alpha C_{T}$ is skew n-normal operator.

Theorem 8. Let $C_{T}$ be the skew n-normal composition operator on a Hilbert space $L^{2}(\mu)$. If $\left(C_{T}\right)^{-1}=C_{T^{-1}}$ then $C_{T^{-1}}$ is skew n-normal composition operator.

Proof. Since $C_{T}$ is skew n-normal composition operator, therefore

$$
C_{T}\left(C_{T}^{*} C_{T}^{n}\right)=\left(C_{T}^{n} C_{T}^{*}\right) C_{T} .
$$

Taking inverse on both sides, we get

$$
C_{T^{-1}}^{n} C_{T^{-1}}^{*} C_{T^{-1}}=C_{T^{-1}} C_{T^{-1}}^{*} C_{T^{-1}}^{n} .
$$

Hence $C_{T^{-1}}$ is skew n-normal composition operator.

A composition operator $C_{S}$ is unitarily equivalent to $C_{T}$ if $C_{S}=U C_{T} U^{*}$ where $U$ is some unitary operator.

Theorem 9. If $C_{T}$ is skew n-normal operator on a Hilbert space $\mathcal{H}$ and $C_{S}$ is unitarily equivalent to $C_{T}$, then $C_{S}$ is skew n-normal operator.

Proof. Since $C_{S}$ is unitarily equivalent to $C_{T}$, then there exists an unitary operator $U$ such that $C_{S}=U C_{T} U^{*}$.

Now

$$
\begin{aligned}
\left(C_{S}^{n} C_{S}^{*}\right) C_{S} & =\left(U C_{T}^{n} U^{*} U C_{T}^{*} U^{*}\right) U C_{T} U^{*} \\
& =U\left(C_{T}^{n} C_{T}^{*}\right) C_{T} U^{*} \\
& =U C_{T}\left(C_{T}^{*} C_{T}^{n}\right) U^{*}
\end{aligned}
$$

because $C_{T}$ is skew n-normal operator. On the other hand

$$
C_{S}\left(C_{S}^{*} C_{S}^{n}\right)=U C_{T} U^{*}\left(U C_{T}^{*} U^{*} U C_{T}^{n} U^{*}\right)=U C_{T}\left(C_{T}^{*} C_{T}^{n}\right) U^{*}
$$

This implies that $\left(C_{S}^{n} C_{S}^{*}\right) C_{S}=C_{S}\left(C_{S}^{*} C_{S}^{n}\right)$. Hence $C_{S}$ is skew n-normal operator.

Theorem 10. Let $C_{S}$ be normal operator and $C_{T}$ be skew n-normal operator.If $C_{S}$ and $C_{T}$ commute, then $C_{S} C_{T}$ is skew n-normal operator. 
Proof. Cosider

$$
\left(\left(C_{S} C_{T}\right)^{n}\left(C_{S} C_{T}\right)^{*}\right) C_{S} C_{T}=\left(C_{S}^{n} C_{T}^{n} C_{T}^{*} C_{S}^{*}\right) C_{S} C_{T}
$$

Since $C_{S}$ is normal operator which commutes with $C_{T}$, then by Fuglede-Putnam theorem, $C_{T}$ commutes with $C_{S}^{*}$ therefore

$$
\begin{aligned}
\left(C_{S}^{n} C_{T}^{n} C_{T}^{*} C_{S}^{*}\right) C_{S} C_{T} & =C_{S}^{n}\left(C_{T}^{n} C_{T}^{*}\right) C_{T} C_{S}^{*} C_{S} \\
& =C_{S}^{n} C_{T}\left(C_{T}^{*} C_{T}^{n}\right) C_{S}^{*} C_{S} \\
& =C_{S} C_{T} C_{T}^{*} C_{S}^{n-1} C_{T}^{n} C_{S}^{*} C_{S} \\
& =C_{S} C_{T}\left(C_{T}^{*} C_{S}^{*} C_{S}^{n-1} C_{T}^{n} C_{S}\right) \\
& =C_{S} C_{T}\left(C_{T}^{*} C_{S}^{*} C_{S}^{n} C_{T}^{n}\right) \\
& =C_{S} C_{T}\left(\left(C_{S} C_{T}\right)^{*}\left(C_{S} C_{T}\right)^{n}\right)
\end{aligned}
$$

Thus $C_{S} C_{T}$ is skew n-normal operator.

Theorem 11. Let $C_{T}$ be the skew n-normal operator, then $C_{T}$ is skew $n+k(n-1)$-normal operator, for every natural number $k$.

Proof. We prove this result by using the method of induction for every natural number $\mathrm{k}$.

(Base case): when $k=1$

$$
\begin{aligned}
\left(C_{T}^{n+(n-1)} C_{T}^{*}\right) C_{T} & =C_{T}^{n-1}\left(C_{T}^{n} C_{T}^{*}\right) C_{T} \\
& =C_{T}^{n-1} C_{T}\left(C_{T}^{*} C_{T}^{n}\right) \\
& =\left(C_{T}^{n} C_{T}^{*}\right) C_{T} C_{T}^{n-1} \\
& =C_{T}\left(C_{T}^{*} C_{T}^{n}\right) C_{T}^{n-1} \\
& =C_{T}\left(C_{T}^{*} C_{T}^{n+(n-1)}\right) .
\end{aligned}
$$

(Inductive step): Suppose the result is true for $n=k$.

To prove the result for $n=k+1$

$$
\begin{aligned}
\left(C_{T}^{n+(k+1)(n-1)} C_{T}^{*}\right) C_{T} & =C_{T}^{n-1}\left(C_{T}^{n+k(n-1)} C_{T}^{*}\right) C_{T} \\
& =C_{T}^{n-1} C_{T}\left(C_{T}^{*} C_{T}^{n+k(n-1)}\right) \\
& =\left(C_{T}^{n} C_{T}^{*}\right) C_{T} C_{T}^{n+k(n-1)-1} \\
& =C_{T}\left(C_{T}^{*} C_{T}^{n}\right) C_{T}^{(n-1)(k+1)} \\
& =C_{T}\left(C_{T}^{*} C_{T}^{n+(k+1)(n-1)}\right) .
\end{aligned}
$$

Therefore $C_{T}$ is skew $n+k(n-1)$-normal operator. 
Theorem 12. Every n-normal composition operator is skew n-normal composition operator.

Proof. Let $C_{T}$ be n-normal operator therefore $\left(C_{T}^{n} C_{T}^{*}\right)=\left(C_{T}^{*} C_{T}^{n}\right)$ and since every n-normal operator is quasi n-normal operator therefore $C_{T}\left(C_{T}^{*} C_{T}^{n}\right)=$ $\left(C_{T}^{*} C_{T}^{n}\right) C_{T}$. Then

$$
C_{T}\left(C_{T}^{*} C_{T}^{n}\right)=\left(C_{T}^{*} C_{T}^{n}\right) C_{T}=\left(C_{T}^{n} C_{T}^{*}\right) C_{T}
$$

Thus $C_{T}$ is skew n-normal operator.

Theorem 13. Every quasi normal composition operator is skew n-normal composition operator.

Proof. Suppose that $C_{T}$ is quasi normal composition operator. Then

$$
C_{T}\left(C_{T}^{*} C_{T}\right)=\left(C_{T}^{*} C_{T}\right) C_{T}
$$

Now

$$
\begin{aligned}
C_{T}^{2}\left(C_{T}^{*} C_{T}\right) & =C_{T}\left[\left(C_{T}^{*} C_{T}\right) C_{T}\right] \\
& =C_{T}\left(C_{T}^{*} C_{T}\right) C_{T} \\
& =\left(C_{T}^{*} C_{T}\right) C_{T}^{2}
\end{aligned}
$$

Similarly $C_{T}^{n-1}$ commutes with $C_{T}^{*} C_{T}$ for every n, so that

$$
\left(C_{T}^{n} C_{T}^{*}\right) C_{T}=C_{T} C_{T}^{n-1}\left(C_{T}^{*} C_{T}\right)=C_{T}\left(C_{T}^{*} C_{T}\right) C_{T}^{n-1}=C_{T} C_{T}^{*} C_{T}^{n} .
$$

Thus $C_{T}$ is skew n-normal composition operator.

\section{3. skew n-Normal Weighted Composition Operator}

Let $(X, \mathcal{A}, \mu)$ be a $\sigma$-finite measure space and $W=W_{\phi, T}$ be the weighted composition operator on $L^{2}(\mu)$ induced by the complex valued function $\phi$ and a measurable transformation $T$. The adjoint $W^{*}$ of $W$ is given by $W^{*} f=$ $h E(\phi f) \circ T^{-1}$ for $f \in L^{2}(\mu)$. We put $\phi_{n}=\phi .(\phi \circ T) .\left(\phi \circ T^{2}\right) \ldots \ldots .\left(\phi \circ T^{n-1}\right)$ where $\mathrm{n}$ be any natural number. For $f \in L^{2}(\mu), W^{n} f=\phi_{n} \cdot f \circ T^{n}$ and $W^{* n} f=$ $h_{n} \cdot E\left(\phi_{n} \cdot f\right) \circ T^{-n}$.

Theorem 14. Let $W$ be a weighted composition operator on $L^{2}(\mu)$. Then the following statements are equivalent:

(i) $W$ is skew n-normal operator.

(ii) $\phi_{n} \cdot h \circ T^{n} E\left(\phi^{2}\right)=\phi . h \circ T E\left(\phi \phi_{n}\right)$. 
Proof. For $f \in L^{2}(\mu)$

$$
\begin{aligned}
\left(W^{n} W^{*}\right) W f & =\left(W^{n} W^{*}\right)(\phi \cdot f \circ T) \\
& =W^{n}\left(h \cdot E\left(\phi^{2} \cdot f \circ T\right) \circ T^{-1}\right) \\
& =\phi_{n}\left(h \cdot E\left(\phi^{2} f\right) \circ T^{n}\right. \\
& =\phi_{n} h \circ T^{n} E\left(\phi^{2}\right) f \circ T^{n} .
\end{aligned}
$$

Also,

$$
\begin{aligned}
W\left(W^{*} W^{n}\right) f & =W W^{*}\left(\phi_{n} \circ T^{n}\right) \\
& =W\left(h E\left(\phi \phi_{n} \cdot f \circ T^{n}\right) \circ T^{-1}\right) \\
& =\phi\left(h \cdot E\left(\phi \phi_{n}\right) f \circ T^{n-1}\right) \circ T \\
& =\phi h \circ T E\left(\phi \phi_{n}\right) f \circ T^{n} .
\end{aligned}
$$

Suppose that $\mathrm{W}$ is a skew n-normal operator then

$$
\left(W^{n} W^{*}\right) W f=W\left(W^{*} W^{n}\right) f \Longleftrightarrow \phi_{n} . h \circ T^{n} E\left(\phi^{2}\right)=\phi \cdot h \circ T E\left(\phi \phi_{n}\right) .
$$

Theorem 15. Let $W$ be a weighted composition operator on $L^{2}(\mu)$. Then the following statements are equivalent:

(i) $W^{*}$ is skew n-normal operator.

(ii) $h E\left(\phi^{2} h_{n} E\left(\phi_{n} f\right)\right)=h_{n} E\left(\phi_{n} \phi h \circ T^{-n+1} E(\phi f)\right)$.

Proof.

$$
\begin{aligned}
W^{*}\left(W W^{* n}\right) f & =W^{*} W\left(h_{n} E\left(\phi_{n} f\right) \circ T^{-n}\right) \\
& =W^{*} \phi\left(h_{n} E\left(\phi_{n} f\right) \circ T^{-n}\right) \circ T \\
& =h E\left(\phi . \phi h_{n} \circ T E\left(\phi_{n} f\right) \circ T^{-n+1}\right) \circ T^{-1} \\
& =h E\left(\phi^{2} h_{n} E\left(\phi_{n} f\right)\right) \circ T^{-n} .
\end{aligned}
$$

Also,

$$
\begin{aligned}
\left(W^{* n} W\right) W^{*} f & =\left(W^{* n} W\right)\left(h E(\phi f) \circ T^{-1}\right) \\
& =W^{* n}(\phi \cdot h \circ T) E(\phi f) \\
& =h_{n} E\left(\phi_{n} \phi \cdot h \circ T E(\phi f)\right) \circ T^{-n} \\
& =h_{n} E\left(\phi_{n} \phi h \circ T^{-n+1} E(\phi f) \circ T^{-n}\right) .
\end{aligned}
$$

If $W^{*}$ is a skew n-normal operator. Then 


$$
\begin{gathered}
W^{*}\left(W W^{* n}\right)=\left(W^{* n} W\right) W^{*} \Longleftrightarrow h E\left(\phi^{2} h_{n} E\left(\phi_{n} f\right)\right)= \\
h_{n} E\left(\phi_{n} \phi h \circ T^{-n+1} E(\phi f)\right) .
\end{gathered}
$$

\section{Acknowledgments}

The authors thank referees for carefully reading manuscript and for the comments and remarks given on it.

The third author is supported by the Senior Research Fellowship of University Grant Commission, New Delhi, India.

\section{References}

[1] Ahmed O. and Ahmed M.S., On the class of n-power quasi-normal operators on Hilbert space, Bull. Math. Anal. Appl., 2 (2011), 213-228.

[2] Alzuraiqi, S.A. and Patel A.B., On n-normal operators, General Math. Notes., 1(2) (2010), 61-73.

[3] Berberian, S.K., Introduction to Hilbert space, Chelsea Publishing Company, New York., (1976), 139-140.

[4] Campbell J. and Jamison J., On some classes of weighted composition operators, Glasgow Math.J., 32 (1990), 82-94.

[5] Dibrell P. and Campbell J.T., Hyponormal powers of composition operators, Proc. Amer. Math. Soc., 102 (1988), 914-918.

[6] Gupta A. and Bhatia N., n-Normal and n-Quasinormal composition operators and weighted composition operators on $L^{2}(\mu)$, Math. Vesnik., 4 (66) (2014) 364-370.

[7] Gupta A. and Bhatia N., On (n,k)- Quasiparanormal weighted composition operators, Int. J. of pure and appl. Math., 1(91) (2014) 23-32.

[8] Harrington D.J. and Whitley R., Seminormal composition operator, J. Oper. Theory., 11 (1981), 125-135.

[9] Panayappan, S., On n-power class (Q) operators, Int. J. of Math. Anal., 6(31) (2012) 1513-1518.

[10] Shaakir L. K. and Abdulwahid E.S., Skew n-normal operators, Aust. J. of basic and appl. sci., 8(16) (2014), 340-344.

[11] Shqipe L., Quasi-normal operators, Int. J. of Math. Anal., 4(47) (2010) 2311-2320.

[12] Singh R.K., Compact and quasinormal composition operators, Proc. Amer. Math. Soc., 45 (1974), 80-82. 